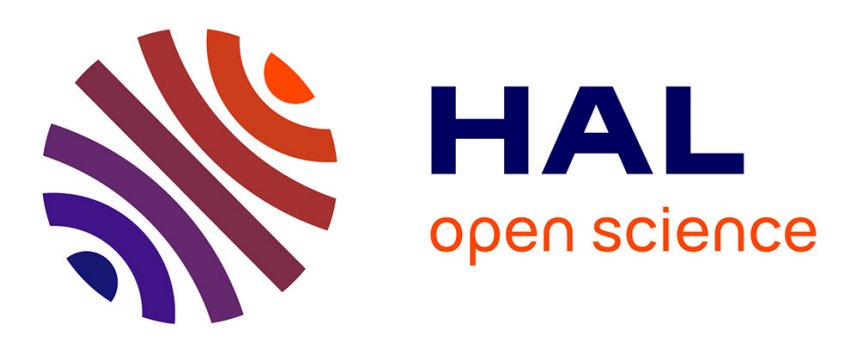

\title{
Dual-Space Adaptive Control of Redundantly Actuated Cable-Driven Parallel Robots
}

\author{
Johann Lamaury, Marc Gouttefarde, Ahmed Chemori, Pierre-Elie Hervé
}

\section{To cite this version:}

Johann Lamaury, Marc Gouttefarde, Ahmed Chemori, Pierre-Elie Hervé. Dual-Space Adaptive Control of Redundantly Actuated Cable-Driven Parallel Robots. IROS: Intelligent RObots and Systems, Nov 2013, Tokyo, Japan. pp.4879-4886, 10.1109/IROS.2013.6697060 lirmm-01221399

\section{HAL Id: lirmm-01221399 \\ https://hal-lirmm.ccsd.cnrs.fr/lirmm-01221399}

Submitted on 28 Oct 2015

HAL is a multi-disciplinary open access archive for the deposit and dissemination of scientific research documents, whether they are published or not. The documents may come from teaching and research institutions in France or abroad, or from public or private research centers.
L'archive ouverte pluridisciplinaire HAL, est destinée au dépôt et à la diffusion de documents scientifiques de niveau recherche, publiés ou non, émanant des établissements d'enseignement et de recherche français ou étrangers, des laboratoires publics ou privés. 


\title{
Dual-Space Adaptive Control of Redundantly Actuated Cable-Driven Parallel Robots
}

\author{
Johann Lamaury $^{1}$, Marc Gouttefarde ${ }^{1}$, Ahmed Chemori ${ }^{1}$ and Pierre-Élie Hervé ${ }^{1}$
}

\begin{abstract}
Cable-driven parallel robots (CDPR) are efficient manipulators able to carry heavy payloads across large workspaces. Therefore, the dynamic parameters such as the mobile platform mass and center of mass location may considerably vary. Without any adaption, the erroneous parametric estimate results in mismatch terms added to the closed-loop system, which may decrease the robot performances. In this paper, we introduce an adaptive dual-space motion control scheme for CDPR. The proposed method aims at increasing the robot tracking performances, while keeping all the cable tensed despite uncertainties and changes in the robot dynamic parameters. Reel-time experimental tests, performed on a large redundantly actuated CDPR prototype, validate the efficiency of the proposed control scheme. These results are compared to those obtained with a non-adaptive dual-space feedforward control scheme.
\end{abstract}

\section{INTRODUCTION}

Cable-driven parallel robots (CDPR) consist of a mobile platform (end-effector) linked to a fixed base through flexible cables. The cable lengths are controlled by means of winches allowing the platform motion control. Cables can be unwound over great lengths such that large workspaces can be obtained. This latter characteristic together with the cable capability of carrying heavy payloads make CDPR particularly well adapted to perform large dimension manipulation or positioning tasks [1]-[4].

In order to drive the platform while controlling all its degrees of freedom (DOF) and to obtain large workspace to footprint ratios, redundant actuation can be used. Several papers proposed redundancy resolution schemes (tension distribution) [5], [6] based on optimization methods. In order to fulfill practical real-time needs, a computationally efficient non-iterative algorithm for tension distribution (TD) was proposed in [7] based on the previous work of [8]. This algorithm works for redundantly actuated CDPR having two more actuators than DOF, i.e. $m=n+2$ where $m$ is the cable number and $n$ the number of DOF of the mobile platform.

The proposed TD algorithm keeps the cable tension vector $\mathbf{t} \in \mathbb{R}^{m}$ between a non-negative minimum value $\mathbf{t}_{\text {min }} \geq$ 0 and a maximum value $\mathbf{t}_{\max }$ (generally induced by the mechanical design). If these constraints are satisfied, then $\mathbf{t}$ is said to be admissible. Note that the non-negativity condition on cable tensions is a key issue in CDPR control. Indeed, cables are flexible links that cannot push on the platform. Consequently, a control action in which a cable is

${ }^{1}$ J. Lamaury, M. Gouttefarde, A. Chemori and Pierre-Élie Hervé are with the Laboratory of Informatics, Robotics and Microelectronics of Montpellier (LIRMM-CNRS-UM2), 161 rue Ada, 34095 Montpellier Cedex 5, France, lamaurydirmm.fr, gouttefardedirmm.fr, chemorialirmm.fr, hervedlirmm.fr required to push on the platform (i.e. with negative tension) is not valid [5]. In this paper, we focus on motion control of a redundantly actuated CDPR where the cable tension constraints are ensured by the TD algorithm introduced in [7].

Compared to the vast literature on robot motion control, few studies deal with CDPR. Kawamura et al. proposed to use a proportional and derivative (PD) controller in joint space [9]. Oh and Agrawal introduced a PD controller based on feedback linearization to asymptotically stabilize the system to the desired pose, taking into account the platform dynamics [5]. In order to compensate this dynamics, computed-torque-like controllers using corrective feedforward terms were also studied. Fang et al. [3] applied a joint space feedforward controller to compensate the motor dynamics. Working in both joint and operational spaces, Vafaei et al. proposed in [10] an integrated controller with two feedforward terms. However, only the platform dynamics is compensated. In order to compensate wind disturbances applied to the cabin of their CDPR, Zi et al. designed a fuzzy proportional-integral controller [11]. In [12], we introduced a dual-space feedforward control scheme in operational space with two feedforward terms to compensate both the platform dynamics and the winch dynamics and friction. In this paper, for performance comparison purposes, the control scheme of [12] is also considered, but with a joint space controller.

The practical implementation of such feedforward control schemes, which are based on inverse dynamics, requires a good knowledge of the system dynamic parameters. Since some of them can be unknown or may vary during the execution of the task, these controllers can provide erroneous inputs, decreasing the tracking and regulation performances. In practice, these drawbacks were observed while experimenting on the CoGiRo suspended CDPR shown in Fig. 2. Some parameter variations, specifically the payload mass, the position of the center of mass (COM) of the loaded mobile platform and uncertainties on the winch friction parameters can significantly affect the global performances of the closedloop system.

In order to handle such parametric uncertainties and variability, adaptive control may be a good solution [13], [14]. Adaptive laws inspired from the controller in [15] have been successfully applied to Delta-like parallel robots [16] with online estimation of the mass of the payload together with the arm and motor inertias. Besides, in [17], Kino et al. used adaptive compensation on a fully constrained CDPR in order to reduce errors on internal forces due to uncertainties in actuator positions. However, dynamic 


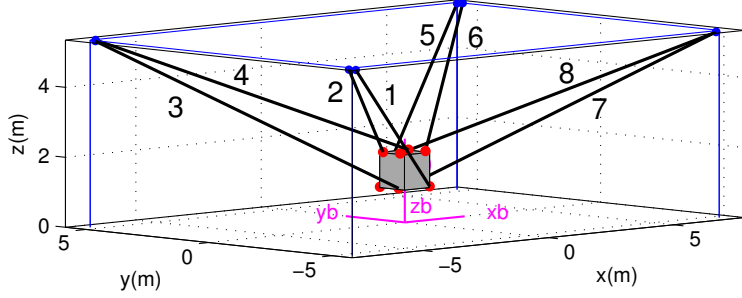

Fig. 1. Schematic view of the 8-cable CoGiRo configuration.

parameter uncertainties were not considered.

The contribution of this paper is an adaptive dual-space control scheme able to perform motion control of redundantly actuated CDPR in presence of parameter uncertainties and variability. Twenty-six parameters are estimated online, namely the loaded (or unloaded) platform mass, the corresponding COM location as well as inertia parameters and the dry and viscous friction coefficients of the winches. The proposed controller only requires the measurements of the actuator rotational positions and speeds. The performances of the proposed adaptive controller are shown through realtime experimental results. The latter, obtained on the CoGiRo suspended CDPR, are compared to those of the non-adaptive dual-space feedforward controller introduced in [12] in order to highlight the performance improvements.

This paper is organized as follows. The dynamic modeling of CDPR is summarized in Section II. The dual-space feedforward control scheme is presented in Section III. Section IV addresses the adaptive controller design while experimental results are presented and discussed in Section V. Finally, conclusions and future works are given in Section VI.

\section{KINEMATIC AND DYNAMIC MODELING}

\section{A. Kinematics}

This work aims at performing an accurate motion control of 6-DOF suspended CDPR equipped with $m$ actuators. The mobile platform pose is defined by an operational space vector $\mathbf{x}_{\phi}=\left[\begin{array}{llll}x y & z & \phi & \theta\end{array}\right]^{T}=\left[\begin{array}{ll}\mathbf{p}^{T} & \mathbf{o}^{T}\end{array}\right]^{T} \in \mathbb{R}^{6}(\mathrm{XYZ}$ Euler angle convention) which contains the position $\mathbf{p}$ and orientation $\mathbf{o}$ of the platform frame $\Re_{p}=\left\{P, x_{p}, y_{p}, z_{p}\right\}$ with respect to the robot base frame $\Re_{b}=\left\{0, x_{b}, y_{b}, z_{b}\right\}$. $P$ is the reference point of the platform. On the CoGiRo prototype, this point is located at the center of the bottom face of the cubic platform, shown in Fig. 2.

The $3 \times 3$ rotation matrix $\mathbf{Q}$ which defines the platform orientation is given by

$\mathbf{Q}(\phi, \theta, \psi)=\left[\begin{array}{ccc}c_{\theta} c_{\psi} & -c_{\theta} s_{\psi} & s_{\theta} \\ c_{\phi} s_{\psi}+s_{\phi} s_{\theta} c_{\psi} & c_{\phi} c_{\psi}-s_{\phi} s_{\theta} s_{\psi} & -s_{\phi} c_{\theta} \\ s_{\phi} s_{\psi}-c_{\phi} s_{\theta} c_{\psi} & s_{\phi} c_{\psi}+c_{\phi} s_{\theta} s_{\psi} & c_{\phi} c_{\theta}\end{array}\right]$,

where $c_{\theta}=\cos (\theta), s_{\psi}=\sin (\psi)$ etc. The joint coordinate vector of motor rotational positions, denoted $\mathbf{q}=\left\{q_{1}, q_{2}, \ldots, q_{m}\right\} \in \mathbb{R}^{m}$, and its time derivative $\dot{\mathbf{q}}$ are assumed to be measured (by means of incremental encoders).

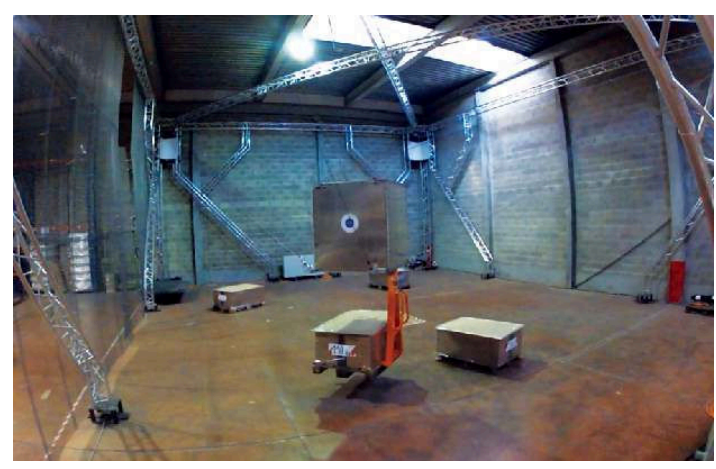

Fig. 2. The LIRMM/Tecnalia COGIRO suspended CDPR prototype.

The relationship between the $i$ th motor angle and the corresponding unwound cable length $l_{i}$ is written as follows

$$
l_{i}= \pm r_{i} q_{i},
$$

where $r_{i}$ accounts for the mechanical transmission reduction ratio and the drum pitch radius of the $i$ th winch. The relationship between the operational and joint space coordinates (inverse kinematics) is as follows

$$
l_{i}=\left\|\left(\mathbf{p}+\mathbf{Q} \mathbf{b}_{i}-\mathbf{a}_{i}\right)\right\|,
$$

where $\mathbf{a}_{i}$ and $\mathbf{b}_{i}$ denote the base drawing point and platform attachment point positions of the $i$ th cable, respectively. The time first derivative of (2) yields

$$
\dot{\mathbf{i}}=\mathbf{R} \dot{\mathbf{q}}=\mathbf{J} \dot{\mathbf{x}},
$$

where $\dot{\mathbf{x}}=\left[\begin{array}{ll}\dot{\mathbf{p}}^{T} & \boldsymbol{\omega}^{T}\end{array}\right]^{T}, \boldsymbol{\omega}$ being the mobile platform angular velocity vector, $\mathbf{i}$ is the time-derivative of $\mathbf{I}=\left[l_{1} \ldots l_{m}\right] \in \mathbb{R}^{m}, \mathbf{J}$ is the $m \times 6$ Jacobian matrix of the CDPR and $\mathbf{R}$ is the diagonal matrix such that $\operatorname{diag}(\mathbf{R})=\left\{r_{1}, r_{2}, \ldots, r_{m}\right\} \in \mathbb{R}^{m}$. The relationship between $\boldsymbol{\omega}$ and $\dot{\mathbf{o}}$, the first time derivative of the vector of Euler angles, is given by

$$
\boldsymbol{\omega}=\left[\begin{array}{c}
\omega_{1} \\
\omega_{2} \\
\omega_{3}
\end{array}\right]=\left[\begin{array}{ccc}
1 & 0 & s \theta \\
0 & c \phi & -s \phi c \theta \\
0 & s \phi & c \phi c \theta
\end{array}\right]\left[\begin{array}{c}
\dot{\phi} \\
\dot{\theta} \\
\dot{\psi}
\end{array}\right]=\mathbf{S} \dot{o} .
$$

By time differentiation, we also have $\dot{\boldsymbol{\omega}}=\dot{\mathbf{S}} \dot{\boldsymbol{o}}+\mathbf{S o}$. The time differentiation of (3) yields

$$
\ddot{\mathbf{I}}=\mathbf{R} \ddot{\mathbf{q}}=\mathbf{J} \ddot{\mathbf{x}}+\mathbf{J} \dot{\mathbf{x}} .
$$

The dynamics of a CDPR may be separated into the dynamics of the mobile parts and the winches. The mobile parts are composed of the mobile platform (equipped with the crane fork in the case of CoGiRo robot shown in Fig.2) and a possible payload.

\section{B. Mobile platform and payload dynamics}

Depending on the task at hand, e.g. if the CDPR performs pick-and-place tasks, the mass, the COM location (called $G$ in the following) and the inertia parameters of the mobile platform (loaded or unloaded) may vary. Consequently, $G$ 


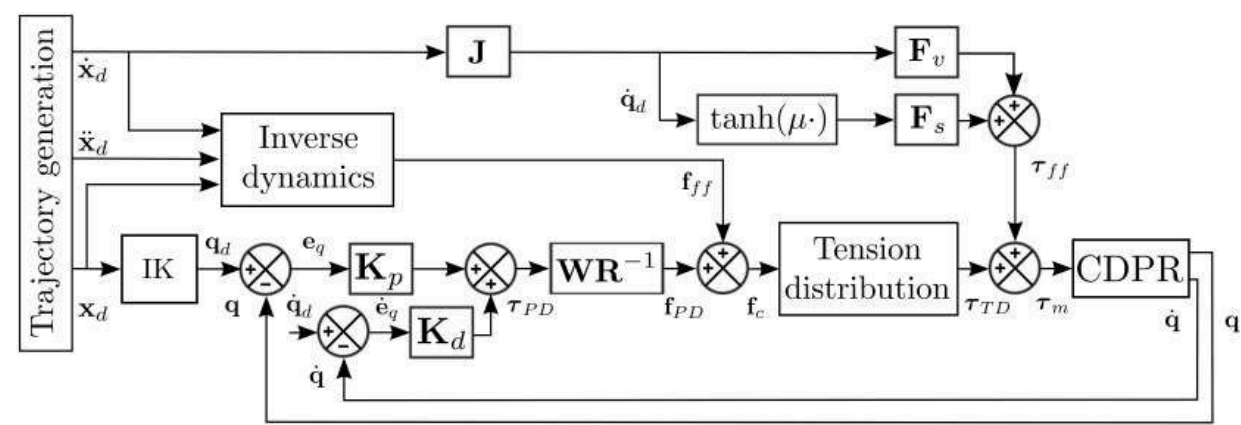

Fig. 3. Dual-space feedforward control scheme with joint space controller.

may not be coincident with $P(G \neq P)$, and the corresponding displacement vector, expressed in the base frame $\Re_{b}$, is denoted by $\mathbf{d}=\overrightarrow{P G}=\left[\begin{array}{lll}u & v & w\end{array}\right]^{T}$. Under this assumption, Newton-Euler equations of motion are expressed by

$$
\mathbf{M}(\mathbf{x}) \ddot{\mathbf{x}}+\mathbf{C}(\mathbf{x}, \dot{\mathbf{x}}) \dot{\mathbf{x}}-\mathbf{f}_{g}(\mathbf{x})=\mathbf{W t},
$$

where $\mathbf{M}$ and $\mathbf{C}$ are $6 \times 6$ matrices defined as

$$
\begin{gathered}
\mathbf{M}(\mathbf{x})=\left[\begin{array}{cc}
m_{t} \mathbf{I}_{3} & -m_{t}[\mathbf{d}] \\
m_{t}[\mathbf{d}] & \mathbf{H}
\end{array}\right], \\
\mathbf{C}(\mathbf{x}, \dot{\mathbf{x}}) \dot{\mathbf{x}}=\left[\begin{array}{c}
m_{t}[\boldsymbol{\omega}][\boldsymbol{\omega}] \mathbf{d} \\
{[\boldsymbol{\omega}] \mathbf{H} \boldsymbol{\omega}}
\end{array}\right] .
\end{gathered}
$$

In these expressions, $m_{t}$ denotes the total mass of the mobile platform with payload and $\mathbf{H}$ is the $3 \times 3$ symmetric matrix $\mathbf{H}=\mathbf{Q I}_{G} \mathbf{Q}^{T}-m_{t}[\mathbf{d}][\mathbf{d}], \mathbf{I}_{G}$ and $\mathbf{H}$ being the mobile platform inertia matrices at the COM $G$ and reference point $P$, respectively. In addition, $[\mathbf{d}]$ and $[\boldsymbol{\omega}]$ denote the $3 \times 3$ skew-symmetric cross product matrices associated to $\mathbf{d}$ and $\omega$, respectively.

In (6), $\mathbf{f}_{g}=m_{t} g\left[\begin{array}{llllll}0 & 0 & -1 & -v & u & 0\end{array}\right]^{T}$ denotes the gravity induced wrench at $P$ and $\mathbf{f}=\mathbf{W t}$ is the wrench applied to the mobile platform by the cables, where $\mathbf{W}$ is the $6 \times m$ wrench matrix than maps the cable tension vector $\mathbf{t} \in \mathbb{R}^{m}$ to the wrench $\mathbf{f} \in \mathbb{R}^{6}$. For CDPR, the wrench matrix is related to the Jacobian matrix by $\mathbf{W}=-\mathbf{J}^{T}$. It is worth noting that the platform dynamics of Eq. (6) is only valid for $\mathbf{t} \geq 0$. This requirement is verified by the proposed control law using a TD algorithm.

Basically, this TD algorithm adds a homogeneous tension solution $\mathbf{t}_{n}$ to the particular tension solution $\mathbf{t}_{p}$ stemming from the Moore-Penrose generalized inverse such that

$$
\mathbf{t}_{\mathrm{TD}}=\mathbf{W}^{+} \mathbf{f}+\mathbf{N} \boldsymbol{\lambda}=\mathbf{t}_{p}+\mathbf{t}_{n},
$$

where $\mathbf{W}^{+}$is the Moore-Penrose generalized inverse of the wrench matrix, $\mathbf{N}=\operatorname{ker}(\mathbf{W})$ is its nullspace and $\boldsymbol{\lambda}$ is an arbitrary vector. For a detailed description of this algorithm, the reader is referred to [7].

\section{Winch dynamics}

The dynamics of the CDPR winches is written as follows

$$
\boldsymbol{\tau}_{m}=\mathbf{I}_{m}(\mathbf{q}) \ddot{\mathbf{q}}+\mathbf{F}_{v} \dot{\mathbf{q}}+\mathbf{F}_{s} \tanh (\mu \dot{\mathbf{q}})+\mathbf{R t},
$$

where $\mathbf{I}_{m} \in \mathbb{R}^{m \times m}$ denotes the inertia matrix of the motors, drums and other rotating parts, $\mathbf{F}_{v} \in \mathbb{R}^{m \times m}$ and $\mathbf{F}_{s} \in \mathbb{R}^{m \times m}$ are the diagonal matrices of viscous and dry friction coefficients, respectively, and $\boldsymbol{\tau}_{m} \in \mathbb{R}^{m}$ is the actuator input torque vector. The approximation introduced by the use of $\tanh (\mu \dot{\mathbf{q}})$ in place of $\operatorname{sgn}(\dot{\mathbf{q}})$ to model dry friction is convenient as it avoids the discontinuities of the latter. In the sequel, $\mu>0$ is set to 1000 in order to approximate the sgn function.

The full robot dynamics can be written in operational space by using (3) and (5), and by substituting $\mathbf{t}$ of (10) into (6). However, both the loaded mobile platform and actuator dynamic equations will be treated separately as implemented in the dual-space feedforward control scheme introduced in the next section.

\section{DUAL-SPACE FEEDFORWARD CONTROL}

The dual-space feedforward control scheme presented in Fig. 3 is designed with a joint space PD controller. As opposed to the operational space PD controller introduced in [12], which uses an operational space controller, its tuning is much simpler in practice. The "inverse dynamics" bloc embeds Eq. (6) in order to compensate the loaded platform dynamics with an operational space feedforward force vector term $\mathbf{f}_{f f}$. The actuator dynamics of Eq. (10) is also compensated by a joint space feedforward torque vector term $\boldsymbol{\tau}_{f f}$. Compared to the other considered torques, in the case of the CoGiRo CDPR considered in Section V, the torques generated by the motor inertia are insignificant due to the winch design, reduction ratio and relatively low motor accelerations. Consequently, $\mathbf{I}_{m}$ will be neglected in the following. However, if necessary, note that the consideration of $\mathbf{I}_{m}$ would represent no particular difficulty. It is also assumed that the mass of the cables is negligible compared to the other involved forces. The actuator input is then given by

$$
\tau_{m}=\tau_{f f}+\boldsymbol{\tau}_{\mathrm{TD}}
$$

where $\boldsymbol{\tau}_{f f}=\mathbf{F}_{v} \dot{\mathbf{q}}_{d}+\mathbf{F}_{s} \tanh \left(\mu \dot{\mathbf{q}}_{d}\right)$ compensates for the winch dynamics and $\boldsymbol{\tau}_{\mathrm{TD}}=\mathbf{R t}_{\mathrm{TD}}$ is the output torque vector of the TD algorithm. $\tau_{\mathrm{TD}}$ is obtained from

$$
\boldsymbol{\tau}_{\mathrm{TD}}=\mathbf{R} \mathbf{W}^{+}\left(\mathbf{f}_{\mathrm{PD}}+\mathbf{f}_{f f}\right)+\mathbf{R} \mathbf{N} \boldsymbol{\lambda},
$$




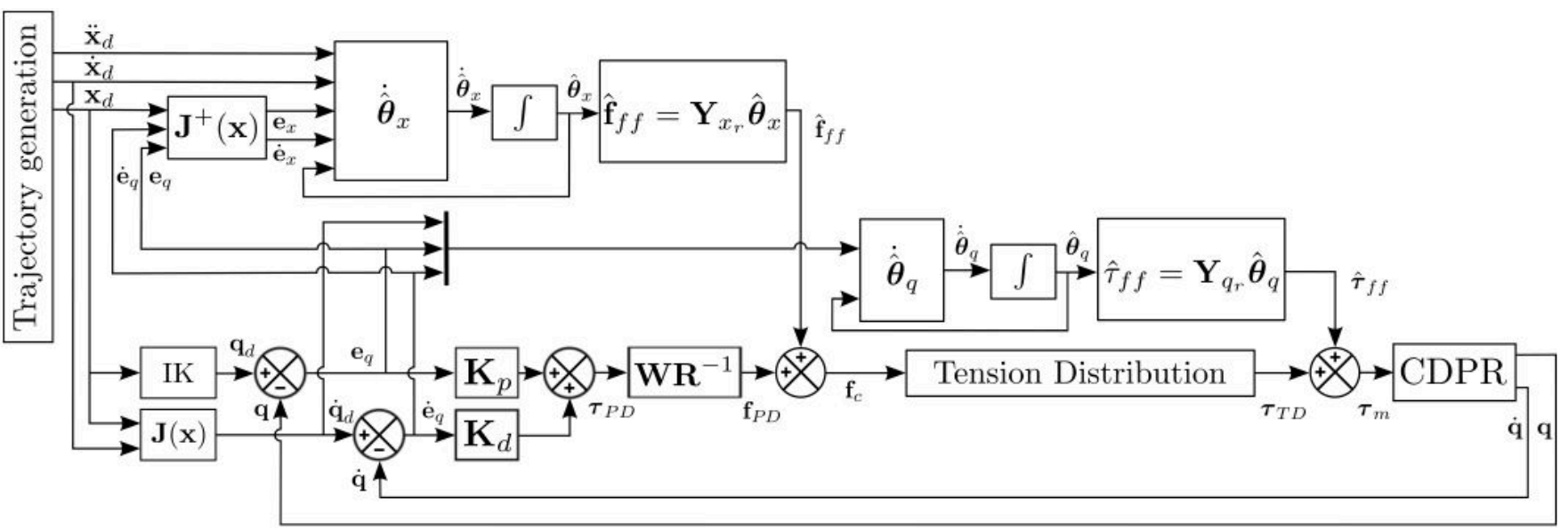

Fig. 4. Dual-space adaptive control scheme with joint space controller.

where $\mathbf{f}_{f f}=\mathbf{M}(\mathbf{x}) \ddot{\mathbf{x}}_{d}+\mathbf{C}(\mathbf{x}, \dot{\mathbf{x}}) \dot{\mathbf{x}}_{d}-\mathbf{f}_{g}(\mathbf{x})$ compensates for the platform dynamics and $\mathbf{f}_{\mathrm{PD}}=\mathbf{W R}^{-1}\left(\mathbf{K}_{p} \mathbf{e}_{q}(t)+\mathbf{K}_{d} \dot{\mathbf{e}}_{q}(t)\right)$ tracks the desired trajectory. Here, $\mathbf{K}_{p}$ and $\mathbf{K}_{d}$ are diagonal positive definite $m \times m$ gain matrices, $\mathbf{e}_{q}$ is the actuator rotational position error vector $\mathbf{e}_{q}=\mathbf{q}_{d}-\mathbf{q}$ and $\dot{\mathbf{e}}_{q}$ the actuator rotational speed error vector $\dot{\mathbf{e}}_{q}=\dot{\mathbf{q}}_{d}-\dot{\mathbf{q}}$. Combining (11) and (12), the overall control law is written as

$$
\begin{aligned}
\boldsymbol{\tau}_{m}= & \boldsymbol{\tau}_{f f}+\mathbf{R} \mathbf{W}^{+} \mathbf{f}_{f f}+\mathbf{R} \mathbf{N} \boldsymbol{\lambda} \\
& +\mathbf{R} \mathbf{W}^{+} \mathbf{W R}^{-1}\left(\mathbf{K}_{p} \mathbf{e}_{q}(t)+\mathbf{K}_{d} \dot{\mathbf{e}}_{q}(t)\right) .
\end{aligned}
$$

In order to keep the platform dynamics valid (i.e. $\mathbf{t} \geq 0$ ), the "Tension distribution" bloc embeds the efficient real-time compatible TD algorithm of [7]. Additionally, the desired accelerations stemming from the "Trajectory generation" bloc are bounded, especially in the $-\mathbf{z}$ direction since the considered CDPR is suspended. However, while experimenting on the CoGiRo redundantly actuated CDPR prototype, it has sometimes been observed that the TD algorithm failed, which means that the forces $\mathbf{f}_{c}$ to be applied by the cables to the platform cannot be performed. This is attributed to model uncertainties. If the inverse dynamics mismatch the CDPR actual dynamics, then feedforward terms provide wrong signals. The PD controller is led to overwork and $\mathbf{f}_{c}$ may become impossible to balance. Such a situation leads to a failure of the TD algorithm which may yield large tracking errors.

In order to solve these modeling uncertainty and variation issues and to improve the global performances of the CDPR, an adaptive dual-space feedforward control scheme able to estimate online the dynamic and friction parameters is proposed in the next section. The new control law is inspired from (13) and allows the feedforward terms $\boldsymbol{\tau}_{f f}$ and $\mathbf{f}_{f f}$ to be adapted during the task.

\section{ADAPTIVE CONTROL DESIGN}

This paper proposes to keep separated the two dynamics equations (6) and (10) in order to apply two corrective feedforward terms in the framework of the dual-space adaptive control scheme shown in Fig. 4. Classical feedforward control relies on a good knowledge of the system parameters. Some of them, such as friction, are very difficult to estimate and may change over time. In the same way, while performing pick-and-place tasks, the total mass of the loaded or unloaded mobile platform together with its COM location can be largely modified. These variations and uncertainties, acting as disturbances on the closed-loop system, may significantly affect the controller performances. In order to deal with this issue, the inverse dynamics is now reformulated with parameter estimations.

\section{A. Joint space adaptive terms}

Eq. (11), can be rewritten as

$$
\boldsymbol{\tau}_{m}=\mathbf{Y}_{q}(\dot{\mathbf{q}}) \boldsymbol{\theta}_{q}+\boldsymbol{\tau}_{T D}
$$

where the winch friction terms are expressed as

$$
\mathbf{F}_{v} \dot{\mathbf{q}}+\mathbf{F}_{s} \tanh (\mu \dot{\mathbf{q}})=\mathbf{Y}_{q}(\dot{\mathbf{q}}) \boldsymbol{\theta}_{q} .
$$

Here, $\mathbf{Y}_{q}$ is the $m \times 2 m$ regression matrix of known functions

$$
\begin{aligned}
\mathbf{Y}_{q}(\dot{\mathbf{q}})= & {\left[\operatorname{diag}\left\{\tanh \left(\mu \dot{\mathbf{q}}_{1}\right), \ldots, \tanh \left(\mu \dot{\mathbf{q}}_{m}\right)\right\},\right.} \\
& \left.\operatorname{diag}\left\{\dot{\mathbf{q}}_{1}, \ldots, \dot{\mathbf{q}}_{m}\right\}\right],
\end{aligned}
$$

and $\boldsymbol{\theta}_{q}$ is the vector of the $2 m$ winch dynamic parameters

$$
\boldsymbol{\theta}_{q}=\left[\begin{array}{llllllll}
f_{s_{1}} & f_{s_{2}} & \cdots & f_{s_{m}} & f_{v_{1}} & f_{v_{2}} & \cdots & f_{v_{m}}
\end{array}\right]^{T},
$$

$m$ of them being the winch dry friction coefficients $f_{s_{i}}$ and the other $m$ being the winch viscous friction coefficients $f_{v_{i}}$.

In the proposed control scheme, $\boldsymbol{\theta}_{q}$ is estimated in the feedforward loop using "reference" velocities, introduced in [14] as

$$
\hat{\boldsymbol{\tau}}_{f f}=\mathbf{Y}_{q_{r}}\left(\dot{\mathbf{q}}_{r}\right) \hat{\boldsymbol{\theta}}_{q}=\hat{\mathbf{F}}_{v} \dot{\mathbf{q}}_{r}+\hat{\mathbf{F}}_{s} \tanh \left(\mu \dot{\mathbf{q}}_{r}\right),
$$

where $\hat{\mathbf{F}}_{v}$ and $\hat{\mathbf{F}}_{s}$ are the estimates of $\mathbf{F}_{v}$ and $\mathbf{F}_{s}$, respectively. The reference velocity vector $\dot{\mathbf{q}}_{r}$ is defined as $\dot{\mathbf{q}}_{r}=\dot{\mathbf{q}}_{d}+\lambda \mathbf{e}_{q}$, where $\lambda$ is a strictly positive scalar defined as $\lambda=\lambda_{0} /\left(1+\left\|\mathbf{e}_{q}\right\|\right)$ [18], $\lambda_{0}$ being a positive constant to be tuned by the user. Using the projection algorithm of Khalil [15], the joint space estimated parameters vary according to the following adaptation law 


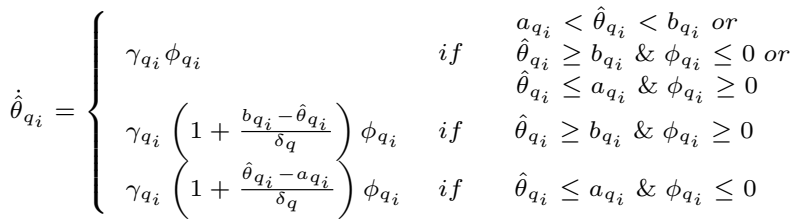

for $i=1 \ldots 2 m$ and where $\delta_{q} \geq 0$. Let us denote $\boldsymbol{\Gamma}_{q}=\operatorname{diag}\left\{\gamma_{q_{1}}, \ldots, \gamma_{q_{2 m}}\right\}$ the positive diagonal matrix of adaptive gains in the joint space. $a_{q_{i}}$ and $b_{q_{i}}$ are the lower and upper bounds on the $i$ th parameter to be estimated, respectively. Furthermore, $\phi_{q}=-\mathbf{Y}_{q r}^{T} \boldsymbol{s}_{q}$, where $\mathbf{s}_{q}$ is the joint space combined error $\mathbf{s}_{q}=\dot{\mathbf{e}}_{q}+\lambda \mathbf{e}_{q}, \phi_{q_{i}}$ being the $i$ th component of $\phi_{q}$. Using (19), for all time $t \geq 0$ and with $a_{q_{i}} \leq \hat{\theta}_{q_{i}}(0) \leq b_{q_{i}}$, all estimated parameters are bounded such that $a_{q_{i}} \leq \hat{\theta}_{q_{i}} \leq b_{q_{i}}$.

\section{B. Operational space adaptive terms}

Let us now consider the tension distribution algorithm output of Eq. (12), which can be rewritten as

$$
\boldsymbol{\tau}_{\mathrm{TD}}=\mathbf{R} \mathbf{W}^{+}\left(\mathbf{f}_{\mathrm{PD}}+\mathbf{Y}_{x}(\mathbf{x}, \dot{\mathbf{x}}, \ddot{\mathbf{x}}) \boldsymbol{\theta}_{x}\right)+\mathbf{R} \mathbf{N} \boldsymbol{\lambda}
$$

where the platform dynamics are expressed as

$$
\mathbf{M}(\mathbf{x}) \ddot{\mathbf{x}}_{d}+\mathbf{C}(\mathbf{x}, \dot{\mathbf{x}}) \dot{\mathbf{x}}_{d}-\mathbf{f}_{g}(\mathbf{x})=\mathbf{Y}_{x}(\mathbf{x}, \dot{\mathbf{x}}, \ddot{\mathbf{x}}) \boldsymbol{\theta}_{x}
$$

$\boldsymbol{\theta}_{x}$ is the vector of the following 10 platform dynamic parameters:

- the total mass $m_{t}$ of the mobile platform and load;

- the three components of $m_{t} \mathbf{d}$, where $\mathbf{d}=[u v w]$ is the COM location as defined in Section II;

- the six different components of the matrix $\mathbf{H}$ :

$$
\mathbf{H}=\left[\begin{array}{ccc}
h_{x x} & -h_{x y} & -h_{x z} \\
-h_{x y} & h_{y y} & -h_{y z} \\
-h_{x z} & -h_{y z} & h_{z z}
\end{array}\right]
$$

Thus, $\boldsymbol{\theta}_{x}$ is defined as follows

$$
\boldsymbol{\theta}_{x}=\left[\begin{array}{llllllllll}
m_{t} & m_{t} u & m_{t} v & m_{t} w & h_{x x} & h_{y y} & h_{z z} & h_{x y} & h_{x z} & h_{y z}
\end{array}\right]^{T} .
$$

The decomposition of the corresponding $6 \times 10$ regressor matrix $\mathbf{Y}_{x}$ as a sum of three $6 \times 10$ matrices gives $\mathbf{Y}_{x}(\dot{\mathbf{x}}, \ddot{\mathbf{x}})=\mathbf{Y}_{x_{1}}(\ddot{\mathbf{x}})+\mathbf{Y}_{x_{2}}(\dot{\mathbf{x}})+\mathbf{Y}_{x_{3}} . \mathbf{Y}_{x_{1}}(\ddot{\mathbf{x}})$ denotes the known functions composing the expression of acceleration forces. It has the following structure

$$
\mathbf{Y}_{x_{1}}(\ddot{\mathbf{x}})=\left[\begin{array}{cccc}
\ddot{\mathbf{p}} & {[\dot{\boldsymbol{\omega}}]} & \mathbf{0}_{3 \times 3} & \mathbf{0}_{3 \times 3} \\
\mathbf{0}_{3 \times 1} & -[\ddot{\mathbf{p}}] & \operatorname{diag}(\dot{\boldsymbol{\omega}}) & \mathbf{E}
\end{array}\right]
$$

where

$$
\mathbf{E}=\left[\begin{array}{ccc}
-\dot{\omega}_{2} & -\dot{\omega}_{3} & 0 \\
-\dot{\omega}_{1} & 0 & -\dot{\omega}_{3} \\
0 & -\dot{\omega}_{1} & -\dot{\omega}_{2}
\end{array}\right]
$$

The expression of $\mathbf{Y}_{x_{2}}(\dot{\mathbf{x}})$, the matrix of known functions of the centrifugal and Coriolis forces, takes the following form

$$
\mathbf{Y}_{x_{2}}(\dot{\mathbf{x}})=\left[\begin{array}{ccc}
\mathbf{0}_{3 \times 1} & \mathbf{A}(\boldsymbol{\omega}) & \mathbf{0}_{3 \times 6} \\
\mathbf{0}_{3 \times 1} & \mathbf{0}_{3 \times 3} & \mathbf{B}(\boldsymbol{\omega})
\end{array}\right]
$$

where $\mathbf{A}$ is the following $3 \times 3$ symmetric matrix

$$
\mathbf{A}=\left[\begin{array}{ccc}
-\left(\omega_{2}^{2}+\omega_{3}^{2}\right) & \omega_{1}^{2} \omega_{2}^{2} & \omega_{1}^{2} \omega_{3}^{2} \\
\omega_{1}^{2} \omega_{2}^{2} & -\left(\omega_{1}^{2}+\omega_{3}^{2}\right) & \omega_{2}^{2} \omega_{3}^{2} \\
\omega_{1}^{2} \omega_{3}^{2} & \omega_{2}^{2} \omega_{3}^{2} & -\left(\omega_{1}^{2}+\omega_{2}^{2}\right)
\end{array}\right]
$$

and $\mathbf{B}$ is a $3 \times 6$ matrix

$$
\mathbf{B}=\left[\begin{array}{cccccc}
0 & -\omega_{2} \omega_{3} & \omega_{2} \omega_{3} & \omega_{1} \omega_{3} & -\omega_{1} \omega_{2} & \left(\omega_{3}^{2}-\omega_{2}^{2}\right) \\
\omega_{1} \omega_{3} & 0 & -\omega_{1} \omega_{3} & -\omega_{2} \omega_{3} & \left(\omega_{1}^{2}-\omega_{3}^{2}\right) & \omega_{1} \omega_{2} \\
-\omega_{1} \omega_{2} & \omega_{1} \omega_{2} & 0 & \left(\omega_{2}^{2}-\omega_{1}^{2}\right) & \omega_{2} \omega_{3} & -\omega_{1} \omega_{3}
\end{array}\right]
$$

The last matrix $\mathbf{Y}_{x_{3}}$ denotes the known functions of the gravity action

$$
\mathbf{Y}_{x_{3}}=\left[\begin{array}{cc}
\mathbf{0}_{2 \times 3} & \mathbf{0}_{2 \times 7} \\
\mathbf{G} & \mathbf{0}_{3 \times 7} \\
\mathbf{0}_{1 \times 3} & \mathbf{0}_{1 \times 7}
\end{array}\right], \quad \mathbf{G}=\left[\begin{array}{ccc}
g & 0 & 0 \\
0 & 0 & g \\
0 & -g & 0
\end{array}\right] .
$$

Using reference velocities and accelerations in the feedforward loop, the platform dynamic parameters are estimated by

$$
\hat{\mathbf{f}}_{f f}=\mathbf{Y}_{x_{r}}\left(\mathbf{x}, \dot{\mathbf{x}}, \dot{\mathbf{x}}_{r}, \ddot{\mathbf{x}}_{r}\right) \hat{\boldsymbol{\theta}}_{x}=\hat{\mathbf{M}}(\mathbf{x}) \ddot{\mathbf{x}}_{r}+\hat{\mathbf{C}}(\mathbf{x}, \dot{\mathbf{x}}) \dot{\mathbf{x}}_{r}-\hat{\mathbf{f}}_{g}(\mathbf{x}),
$$

where $\hat{\mathbf{M}}, \hat{\mathbf{C}}$ and $\hat{\mathbf{f}}_{g}$ are the estimates of $\mathbf{M}, \mathbf{C}$ and $\mathbf{f}_{g}$, respectively. The reference velocities $\dot{\mathbf{x}}_{r}$ and accelerations $\ddot{\mathbf{x}}_{r}$ are defined as $\dot{\mathbf{x}}_{r}=\dot{\mathbf{x}}_{d}+\lambda \mathbf{e}_{x}$ and $\ddot{\mathbf{x}}_{r}=\ddot{\mathbf{x}}_{d}+\lambda \dot{\mathbf{e}}_{x}$, respectively, where $\mathbf{e}_{x}$ and $\dot{\mathbf{e}}_{x}$ denote the position and velocity operational space errors. The adaptive law updating the 10 operational space parameters is given by

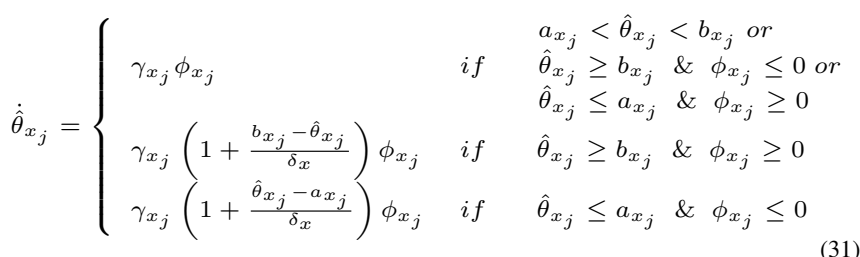

for $j=1 \ldots 10$ and where $\delta_{x} \geq 0$. Let us denote $\boldsymbol{\Gamma}_{x}=\operatorname{diag}\left\{\gamma_{x_{1}}, \ldots, \gamma_{x_{10}}\right\}$ the positive diagonal matrix of adaptive gains in the operational space. $a_{x_{j}}$ and $b_{x_{j}}$ are the lower and upper bounds on the $j$ th parameter to be estimated, respectively. Furthermore, $\phi_{x}=-\mathbf{Y}_{x r}^{T} \boldsymbol{s}_{x}$, where $\mathbf{s}_{x}$ is the operational space combined error $\mathbf{s}_{x}=\dot{\mathbf{e}}_{x}+\lambda \mathbf{e}_{x}=\mathbf{J}^{+} \mathbf{s}_{q}, \phi_{x_{j}}$ being the $j$ th component of $\phi_{x}$. Again, using (19), $\forall t \geq 0$ and with $a_{x_{i}} \leq \hat{\theta}_{x_{i}}(0) \leq b_{x_{i}}$, all these parameters are bounded such that $a_{x_{i}} \leq \hat{\theta}_{x_{i}} \leq b_{x_{i}}$. All bounds, $\delta$ values, adaption gains $\gamma$ and $\lambda_{0}$ must be defined by the user.

\section{Overall control law}

Finally, combining Eq. (14) and (20) while substituting the adaptive terms of Eq. (18) and (30), the proposed adaptive control law is written as

$$
\begin{aligned}
& \boldsymbol{\tau}_{m}=\mathbf{Y}_{q_{r}}\left(\dot{\mathbf{q}}_{r}\right) \hat{\boldsymbol{\theta}}_{q}+\mathbf{R} \mathbf{W}^{+} \mathbf{Y}_{x_{r}}\left(\mathbf{x}, \dot{\mathbf{x}}, \dot{\mathbf{x}}_{r}, \ddot{\mathbf{x}}_{r}\right) \hat{\boldsymbol{\theta}}_{x} \\
& +\mathbf{R} \mathbf{N} \boldsymbol{\lambda}+\mathbf{R} \mathbf{W}^{+} \mathbf{W} \mathbf{R}^{-1}\left(\mathbf{K}_{p} \mathbf{e}_{q}(t)+\mathbf{K}_{d} \dot{\mathbf{e}}_{q}(t)\right) .
\end{aligned}
$$

The dual-space adaptive control scheme shown in Fig. 4 has been implemented in the CoGiRo CDPR control system. Experimental results are given in the following section. 


\section{REAL-TIME EXPERIMENTAL RESULTS}

\section{A. CoGiRo prototype}

The CoGiRo CDPR prototype shown in Fig. 2 has the following main characteristics:

- $15 \mathrm{~m} \times 11 \mathrm{~m} \times 6 \mathrm{~m}(L \times l \times h)$ overall dimensions with a potential workspace of $677 \mathrm{~m}^{3}$;

- Cubic mobile platform of side length $1 \mathrm{~m}$ in suspended configuration equipped with a crane fork. Its total mass is about $m_{p}=93 \mathrm{~kg}$;

- 6 degrees of freedom (DOF) mobile platform;

- $m=8$ actuators so that the robot has 2 degrees of actuation redundancy;

- At least $300 \mathrm{~kg}$ payload capability over all the workspace and $500 \mathrm{~kg}$ as long as the platform is not too close to the workspace boundary;

- $\mathbf{t}_{\min }=0 \mathrm{~N}$ in order to respect the non-negativity constraint on cable tensions and $\mathbf{t}_{\max }=5000 \mathrm{~N}$ according to the maximum capabilities of the force sensors.

- The motor rotational positions and speeds are obtained by means of incremental absolute encoders.

- The reference point $P$ is located at the center of the bottom face of the platform.

As shown in Fig. 1 and Fig. 2, CoGiRo is a suspended CDPR since all the cable drawing points are located near the top of the base frame. In contrast to fully constrained CDPR for which there exists at least one mobile platform pose having force-closure [9], gravity is here used to help keeping the cables tensed.

This section reports some experimental results of the implementation in ANSI $\mathrm{C}$ code in the control system of CoGiRo CDPR of the control scheme proposed in Section IV with a $2 \mathrm{kHz}$ sampling frequency. The control system programming interface is B\&R Automation Studio.

\section{B. Scenario}

The tracking and adaptive performances will be evaluated along the pick-and-place trajectory of the reference point $P$ shown in Fig. 5. The mobile platform, equipped with a crane fork, starts from point 0 . It goes to point $A$ and moves forward to pick up a $110 \mathrm{~kg}$ pallet $(\Delta m=120 \%)$ at point $B$ at time $t_{1}=19 \mathrm{~s}$. The pallet is then displaced and dropped off at point $C$ at time $t_{2}=40 \mathrm{~s}$ before a backward move of the platform to point $D$. In a last step, the mobile platform comes back to its initial position 0 . The paper is accompanied by a video, showing the CoGiRo CDPR performing this pickand-place task via the proposed adaptive control scheme.

In this section, this pick-and-place task will be firstly performed with a feedforward-free control scheme (i.e. controller of Fig. 3 with $\mathbf{f}_{f f}=\mathbf{0}$ and $\boldsymbol{\tau}_{f f}=\mathbf{0}$ ), and secondly using the dual-space feedforward control scheme shown in Fig 3. Finally, the dual-space adaptive control scheme shown in Fig. 4 is applied. The performances of the proposed control schemes are quantified by comparison of the maximum value $\epsilon_{\max }$ and root mean square (RMS) value $\epsilon_{r m s}$ of both the position tracking error (PTE) and orientation tracking error (OTE) in the operational space.

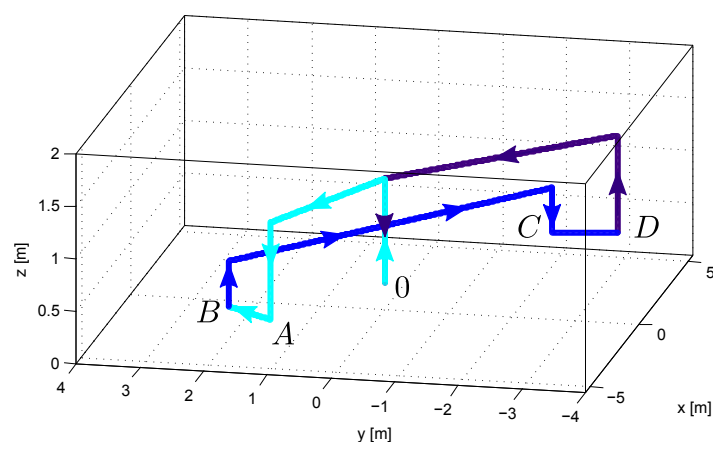

Fig. 5. Pick-and-place trajectory performing a $110 \mathrm{~kg}$ pallet lifting.

TABLE I

MAXIMUM AND RMS TRACKING ERRORS OF THE DIFFERENT CONTROLLER CONFIGURATIONS.

\begin{tabular}{|l||l|l|l|}
\hline error type & $\begin{array}{l}\text { no feedfor- } \\
\text { ward }\end{array}$ & $\begin{array}{l}\text { feedforward } \\
\text { controller }\end{array}$ & $\begin{array}{l}\text { adaptive } \\
\text { controller }\end{array}$ \\
\hline \hline maximum PTE & $37 \mathrm{~mm}$ & $24 \mathrm{~mm}$ & $19 \mathrm{~mm}$ \\
\hline maximum OTE & $7.8 e^{-3} \mathrm{rad}$ & $4.1 e^{-3} \mathrm{rad}$ & $3.9 e^{-3} \mathrm{rad}$ \\
\hline RMS PTE & $20.24 \mathrm{~mm}$ & $7.02 \mathrm{~mm}$ & $0.71 \mathrm{~mm}$ \\
\hline RMS OTE & $2.1 e^{-3} \mathrm{rad}$ & $1.2 e^{-3} \mathrm{rad}$ & $8.7 e^{-5} \mathrm{rad}$ \\
\hline
\end{tabular}

Note that CoGiRo is here not equipped with external measurement system able to provide the current operational pose $\mathbf{x}$ and velocity $\dot{\mathbf{x}}$ of the mobile platform. In order to get the operational tracking errors $\mathbf{e}_{x}$ and $\dot{\mathbf{e}}_{x}$, the joint tracking errors $\mathbf{e}_{q}$ and $\dot{\mathbf{e}}_{q}$ are converted by means of the pseudo-inverse of the Jacobian matrix. Indeed, from (3) we write $\dot{\mathbf{e}}_{x}=\mathbf{J}^{+} \mathbf{R} \dot{\mathbf{e}}_{q}$. Furthermore, the sampling period being small ( $\Delta t$ is equal to $0.5 \mathrm{~ms}$ ), we can write $\mathbf{e}_{q}=\dot{\mathbf{q}} \Delta t$ and $\mathbf{e}_{x}=\dot{\mathbf{x}} \Delta t$ and, consequently, $\mathbf{e}_{x}=\mathbf{J}^{+} \mathbf{R e}_{q}$.

\section{Dual-space feedforward control results}

In the first case, the proposed scenario is applied on CoGiRo without any dynamic compensations $\left(\mathbf{f}_{f f}=\mathbf{0}\right.$ and $\left.\boldsymbol{\tau}_{f f}=\mathbf{0}\right)$. The controller gains are tuned to be $\operatorname{diag}\left(\mathbf{K}_{p}\right)=$ $\{30,30, \ldots, 30\}$ and $\operatorname{diag}\left(\mathbf{K}_{d}\right)=\{1,1, \ldots, 1\}$. The position and orientation maximum tracking error values and
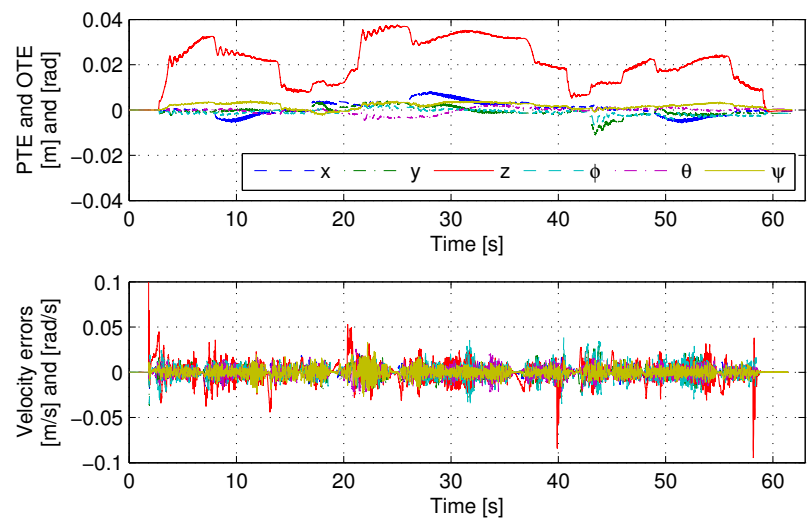

Fig. 6. Position and velocity tracking errors in operational space without feedforward compensation terms. 
RMS error values are large, as shown in Table I. The obtained operational tracking errors (calculated from articular tracking errors, see Section V-B) are depicted in Fig 6. As shown in the latter figure, the most important tracking error is along the $z$-axis: As the mass of the mobile platform and payload is not compensated, primarily, the error remains superior to 0 , with a maximum value of $3.7 \mathrm{~cm}$.

In the second case, the dual-space feedforward control scheme is applied to CoGiRo in order to perform the pickand-place task defined in Section V-B. The controller gain tuning of the previous case is retained. The sum of the mobile platform and the crane fork masses is set to $93 \mathrm{~kg}$. The corresponding COM location and the inertia parameters have been computed using a CAD model. Dry and viscous friction coefficients were estimated empirically (not without difficulties). The obtained operational tracking errors are depicted in Fig 7. Compared to the previous case, the maximum tracking position error is reduced by $50 \%$ and the RMS position error is almost divided by 3 . The position tracking error remains close to 0 when the platform is unloaded. However, when the payload is lifted (between $t_{1}=19 \mathrm{~s}$ and $t_{2}=40 \mathrm{~s}$ ), the error along the $\mathbf{z}$-axis reaches $2.4 \mathrm{~cm}$. Table I shows the maximum and RMS tracking error values in position and orientation of the platform. In order to further improve the tracking performances, we now apply the proposed adaptive control scheme introduced in Section IV.

\section{Dual-space adaptive control results}

CoGiRo is equipped with 8 actuators so that $2 m=16$ parameters are estimated in the joint space. The following table summarizes the whole adaptive control settings.

The control input signals are shown in Fig. 8. As the motor maximum torques are about $280 \mathrm{Nm}$, these signals remain far from the limits of the actuators. The obtained operational tracking errors are depicted in Fig.9. As shown, when the payload is picked at time $t_{1}=19 \mathrm{~s}$ the error along the $z$-axis suddently raises so that the maximum PTE is barely decreased, but it then returns close to 0 due to the adaption process. Consequently, compared to the dual-space feedforward controller, the mean PTE between $t_{1}=19 \mathrm{~s}$ and

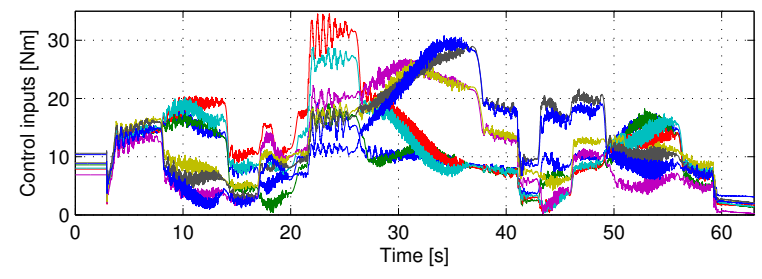

Fig. 8. Control input signals.

TABLE II

ADAPTIVE CONTROLLER TUNING.

\begin{tabular}{|c|c|}
\hline Adaptive gains $\boldsymbol{\Gamma}_{q}$ & $\operatorname{diag}\left(\boldsymbol{\Gamma}_{q}\right)=\left\{0.5, \ldots, 0.5,1 e^{-4}, \ldots, 1 e^{-4}\right\}$ \\
\hline Adaptive gains $\boldsymbol{\Gamma}_{x}$ & $\operatorname{diag}\left(\boldsymbol{\Gamma}_{x}\right)=\left\{120,10,10,10,1 e^{3} \ldots, 1 e^{3}\right\}$ \\
\hline $\mathbf{a}_{q}$ & {$\left[\begin{array}{lllll}0 & 0 & \ldots & 0\end{array}\right]$} \\
\hline $\mathbf{b}_{q}$ & {$\left[\begin{array}{lllllll}5 & \ldots & 5 & 0.03 & \ldots & 0.03\end{array}\right]$} \\
\hline $\mathbf{a}_{x}$ & {$\left[\begin{array}{lllllll}0 & -250 & -250 & -250 & -150 & \ldots & -150\end{array}\right]$} \\
\hline $\mathbf{b}_{x}$ & {$\left[\begin{array}{llllllll}600 & 250 & 250 & 50 & 150 & \ldots & 150\end{array}\right]$} \\
\hline$\lambda_{0}$ & 10 \\
\hline$\delta_{q}$ & 0.001 \\
\hline$\delta_{x}$ & 0.1 \\
\hline Proportional gains & $\operatorname{diag}\left(\mathbf{K}_{p}\right)=\{30,30, \ldots, 30\}$ \\
\hline Derivate gains & $\operatorname{diag}\left(\mathbf{K}_{v}\right)=\{1,1, \ldots, 1\}$ \\
\hline
\end{tabular}

$t_{2}=40 \mathrm{~s}$ is strongly decreased. As reported in Table I, the RMS PTE and RMS OTE are divided by 10 and 14, respectively. The parameters estimations are depicted in Fig. 10. The evolution of the adapted mass parameter indicates that the unloaded platform is estimated to $150 \mathrm{~kg}$ instead of the expected $93 \mathrm{~kg}$. This may be due to non-modeled frictions induced by the drawing point eyelets. However, the increase of the $110 \mathrm{~kg}$ mass between $t_{1}$ and $t_{2}$ is correctly estimated. In Fig. 10, $\delta$ denotes the COM location with respect to the reference point $P$ expressed in the mobile frame $\Re_{p}$. The mass and COM location decrease at the very end of the estimations because the platform is put down on the ground, back at the initial point 0 .

Note that in the three cases of Fig. 6, Fig. 7 and Fig. 9, the velocity tracking errors are similar.

These experiments show clearly the effectiveness of the proposed adaptive controller as well as the effect of the
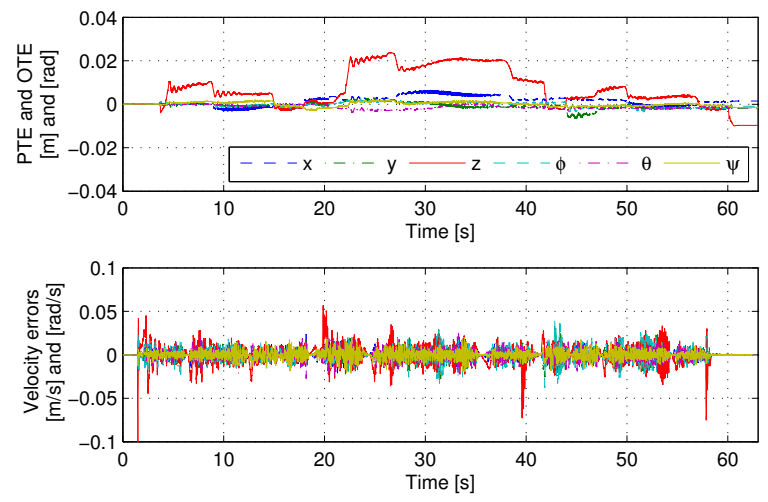

Fig. 7. Position and velocity tracking errors in operational space with the non-adaptive dual-space feedforward controller.
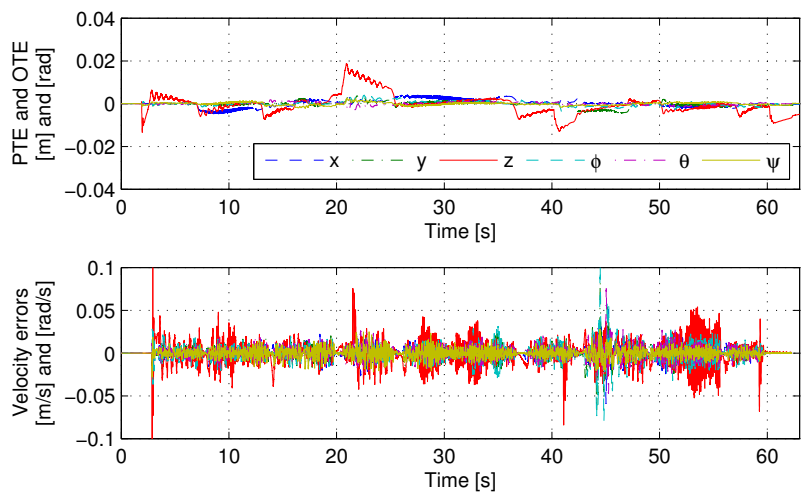

Fig. 9. Position and velocity tracking errors in operational space with the adaptive dual-space feedforward controller. 

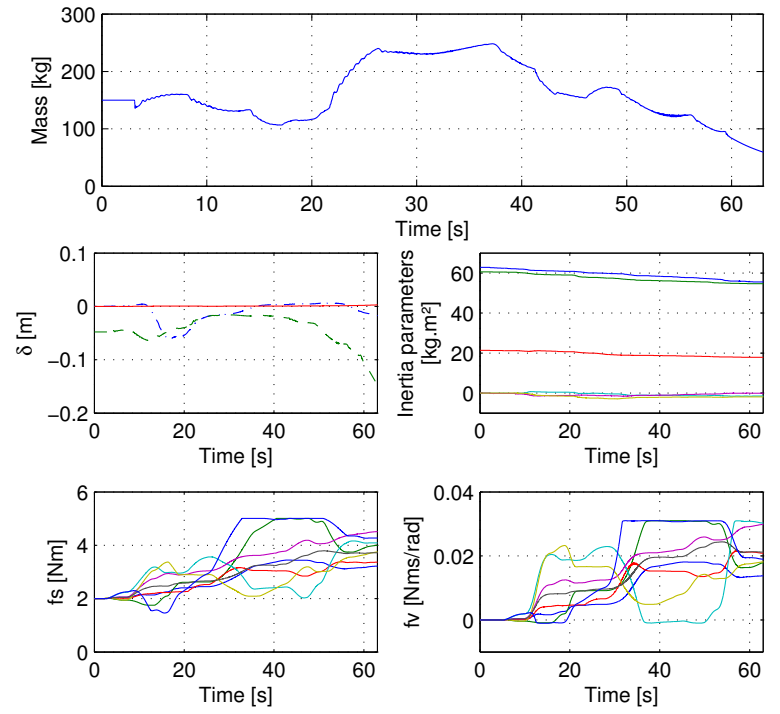

Fig. 10. Parameter estimations.

feedforwards in the performance of the closed-loop controlled system. Ongoing works aim at further improving the controller performances. Notably, as illustrated in Fig. 10, the reactivity of the parameter online estimations could be improved, especially those of the friction coefficients, which should in turn reduce the tracking error of Fig.9.

\section{CONCLUSIONS}

The main contribution of this paper is a dual-space adaptive controller applied to redundantly actuated CDPR. A dual-space feedforward control scheme with a joint space controller was first introduced. An adaptive version of this controller was then proposed to improve its tracking performances. As the former is prone to parametric uncertainties and variations, the adaptive online estimation of the mobile platform and actuator dynamic parameters resulted in better tracking performances. In our experiments on a redundant CDPR, the proposed adaptive controller proved to be stable. Part of our future works will be dedicated to a rigorous stability analysis of the resulting closed-loop system.

\section{ACKNOWLEDGMENT}

The financial support of the ANR (grant 2009 SEGI 018 01) and of the Région Languedoc-Roussillon (grants 115217 and 120218) are greatly acknowledged.

\section{REFERENCES}

[1] J. Albus, R. Bostelman, and N. Dagalakis, "The NIST Robocrane," Jour. of Robotic Systems, vol. 10, no. 2, pp. 709-724, 1993.

[2] W.-J. Shiang, D. Cannon, and J. Gorman, "Optimal Force Distribution Applied to a Robotic Crane with Flexible Cables," in IEEE Int. Conf. on Robotics and Automation, San Francisco, CA, USA, 2000, pp. 1948-1954.

[3] S. Fang, D. Franitza, M. Torlo, F. Bekes, and M. Hiller, "Motion Control of a Tendon-Based Parallel Tension Distribution," IEEE/ASME Trans. on Mechatronics, vol. 9, no. 3, pp. 561-568, 2004.

[4] C. Lambert, M. Nahon, and D. Chalmers, "Implementation of an Aerostat Positioning System with Cable Control," Trans. on Mechatronics, vol. 12, no. 1, pp. 32-40, 2007.

[5] S.-R. Oh and S. K. Agrawal, "Cable Suspended Planar Robots With Redundant Cables: Controllers with Positive Tensions," IEEE Trans. on Robotics, vol. 21, no. 3, pp. 457-465, 2005.

[6] P. H. Borgstrom, B. L. Jordan, G. S. Sukhatme, M. A. Batalin, and W. J. Kaiser, "Rapid Computation of Optimally Safe Tension Distributions for Parallel Cable-Driven Robots," IEEE Trans. on Robotics, vol. 25 , no. 6, pp. 1271-1281, Dec. 2009.

[7] J. Lamaury and M. Gouttefarde, "A Tension Distribution Method with Improved Computational Efficiency," in First Int. Conf. on CableDriven Parallel Robots, T. Bruckmann and A. Pott, Eds., Stuttgart, Germany, 2012, pp. 71-85.

[8] L. Mikelsons, T. Bruckmann, M. Hiller, and D. Schramm, "A RealTime Capable Force Calculation Algorithm for Redundant TendonBased Parallel Manipulators," in IEEE Int. Conf. on Robotics and Automation, Pasadena, CA, USA, May 2008.

[9] S. Kawamura, H. Kino, and C. Won, "High-Speed Manipulation by Using Parallel Wire-Driven Robots," Robotica, vol. 18, pp. 13-21, 2000 .

[10] A. Vafaei, M. M. Aref, and H. D. Taghirad, "Integrated Controller For An Over Constrained Cable Driven Parallel Manipulator : KNTU CDRPM," in IEEE Int. Conf. on Robotics and Automation, Anchorage, Alaska, USA, 2010.

[11] B. Zi, B. Duan, J. Du, and H. Bao, "Dynamic modeling and active control of a cable-suspended parallel robot," Mechatronics, vol. 18, no. 1, pp. 1-12, Feb. 2008.

[12] J. Lamaury and M. Gouttefarde, "Control of a Large Redondantly Actuated Cable-Suspended Parallel Robot," in IEEE Int. Conf. on Robotics and Automation, Karlsruhe, Germany, 2013.

[13] J. J. Craig, P. Hsu, and S. S. Sastry, "Adaptative Control of Mechanical Manipulators," The Int. Jour. of Robotics Research, pp. 190-195, 1986.

[14] J.-J. E. Slotine and W. Li, "On the Adaptive Control of Robot Manipulators," The Int. Jour. of Robotics Research, vol. 6, no. 3, pp. 49-59, Sep. 1987.

[15] K. W. Lee and H. K. Khalil, "Adaptive Output Feedback Control of Robot Manipulators Using High-Gain Observer," Int. Jour. Control, vol. 67, no. 6, pp. 869-886, 1997.

[16] G. S. Natal, A. Chemori, and F. Pierrot, "Dual-Space Adaptive Control of Redundantly Actuated Parallel Manipulators for Extremely Fast Operations With Load Changes," in IEEE Int. Conf. on Robotics and Automation, St. Paul, MN, USA, 2012.

[17] H. Kino, T. Yahiro, T. Fumiaki, and T. Morizono, "Robust PD Control Using Adaptative Compensation for Completely Restrained ParallelWire Driven Robots," in IEEE Trans. on Robotics, vol. 23, no. 4, 2007 , pp. 803-812.

[18] H. Berghuis, R. Ortega, and H. Nijmeijer, "A Robust Adaptive Robot Controller," IEEE Trans. on Robotics and Automation, vol. 9, no. 6, pp. 825-830, 1993. 